\title{
Mathematical description of the increase in selectivity of an an- ion-exchange membrane due to its modification with a per- fluorosulfonated ionomer
}

\author{
Anton Kozmai, Natalia Pismenskaya, Victor Nikonenko*
}

Membrane institute, Kuban State University, 149, Stavropolskaya Str., Krasnodar, 350040, Russian Federation;
kozmay@yandex.ru (A.K.); n_pismen@mail.ru (N.P.); v_nikonenko@mail.ru (V.N.)
* Correspondence: v_nikonenko@mail.ru; Tel.: +7-918-41-45-816

\begin{abstract}
Ion-exchange membranes (IEMs) are widely used in desalination, waste water treatment, food, energy production and other applications. There is a strong demand for cost-effective IEMs characterized by high selective transport of ions of a certain sign of charge. In this paper, we simulate the experimental results of V. Sarapulova et al. (IJMS 2021) on the modification of an inexpensive anion-exchange membrane (CJMA-7, Hefei Chemjoy Polymer Materials Co. Ltd., China) with a perfluorosulfonated ionomer (PFSI). The modification was made in several stages including keeping the membrane at a low temperature, applying a PFSI solution on its surface, and subsequent drying it at an elevated temperature. We apply the known microheterogeneous model with some new amendments to simulate each stage of the membrane modification. It has been shown that the PFSI film formed on the membrane-substrate does not affect significantly its properties due to the small thickness of the film $(\approx 4 \mu \mathrm{m})$ and similar properties of the film and substrate. The main effect is caused by the fact that PFSI material "clogs" the macropores of the CJMA-7 membrane, thereby blocking the transport of coions through the membrane. In this case, the membrane microporous gel phase, which has a high selectivity to counterions, remains the primary pathway for both counterions and coions. Due to the above modification of the CJMA-7 membrane, the coion $\left(\mathrm{Na}^{+}\right)$ transport number in the membrane equilibrated with $1 \mathrm{M} \mathrm{NaCl}$ solution decreased from 0.11 to 0.03 . Thus, the modified membrane becomes comparable in its transport characteristics with more expensive IEMs available on the market.
\end{abstract}

Keywords: ion-exchange membrane; electric conductivity; diffusion permeability; permselectivity; structure-properties relationship; modification; microheterogeneous model

\section{Introduction}

The selectivity of ion-exchange membranes (IEM) in relation to counterion transport is the main functional property of this type of membranes. This property determines the possibility of desalination and concentration of electrolyte solutions by the electrodialysis method [1-3] . A good counterion permselectivity is needed for numerous applications of IEMs: for processing solutions in the agro-food industry, in particular, for the production of fertilizers [4,5], in fuel cells [6-9], flow batteries [9-11] and other processes [12-15].

Commercial IEMs, depending on the manufacturing method and structure, are divided into two large groups: homogeneous and heterogeneous membranes. The competition between these two types of IEM has been lasting for more than half a century [16,17]. In general, the electrochemical properties of homogeneous membranes, including the selectivity to counterion transport, are superior to those of heterogeneous membranes, the main advantage of which is low production cost and higher stability during operation.

The technology of IEM production is constantly being improved. However, the development of new types of membranes is rather costly, therefore, despite their diversity, 
their number remains limited [4] . Modification of membranes is carried out using a small amount of modifying agents and, usually, leads to a slight increase in their cost [18].

Modification of membranes is widely used in membrane technology. The reverse osmosis method became widely used only after the illustrious innovation of Loeb and Surirajan, who in the early 60s of the last century proposed to produce membranes with a thin active surface layer [19]. As for IEMs, the use of membranes consisting of a substrate and an active layer is less common, if bipolar membranes are not taken into account. Apparently, one of the first who used such a technique were Kedem et al. [20]. They covered heterogeneous IEM with a thin layer of a homogeneous ion-conducting film to "suppress concentration polarization". As a result, the limiting current density increased, and the potential drop across the membrane at a given current density decreased. A similar technique was used in Refs. [21,22]. In addition to the above effects, membranes with a homogenized surface showed a significant increase in fouling resistance: the growth rate of precipitation on the membrane surface in solutions containing hardness ions significantly decreased or was completely absent [23].

A thin active layer on an IEM is also used to increase the specific selectivity of ion transport across a composite membrane with respect to singly charged ions [24-26] or with respect to nitrates as compared to chlorides [27] The mechanisms of the modifying layer formation in the process of multilayer membranes manufacturing, as well as the reasons for the selectivity of these membranes to individual ions are considered in Refs. $[24,25]$. However, more often, an empirical approach is used, where the modifying layer nature and thickness are determined by the trial-and-error method [28,29]. The deposition on the membrane surface of a thin layer with fixed ions charged opposite to the charge of the substrate membrane makes it possible to obtain asymmetric bipolar membranes. By adjusting the fixed ions density and their nature in a thin layer, as well as the thickness of this layer, it is possible to control the rate of $\mathrm{H}^{+}$and $\mathrm{OH}^{-}$ions generation at the bipolar boundary, while retaining the function of the membrane for desalination and concentration of electrolytes [30-32].

Although there are numerous papers devoted to the development of monovalention-selective membranes including the method of modification [12,33-38], there are only a few papers where this method is used to improve the counterion permselectivity [3941]. Sarapulova et al. [39], in order to improve the counterion permselectivity, relied on a known fact that coions mainly pass through the membrane within relatively large (macro)pores [39,42,43]. Therefore, the idea of their modification was to clog the macropores with a homogeneous microporous ion-exchange material. After the modification of a homogeneous CJMA-7 membrane, the coion transport number decreased in 2.5 times (in $1.0 \mathrm{M} \mathrm{NaCl}$ solution). This interesting and promising result stimulates theoretical research to better understand the effect and assess the prospects of the applied approach.

To describe the effect of clogging the macropores, the applied model must take into account the ion transport in the pores of different size - not only in the macropores, but in micro- and mesopores also. In the literature, two main types of models, which are applied to describe the ion and water transport in IEM, can be distinguished: 1) "solutiondiffusion" [44-47] and 2) "pore-flow" models [48,49]. Models of the first type do not allow one to determine an explicit relationship between the parameters of the membrane structure and transport coefficients. However, this relationship can be established, when considering the membrane as a multiphase microheterogeneous system (effective-medium approach) [50]. Models of the second type simulate the ion and water transport directly in a membrane pore. However, the complexity of the mathematical description allows considering only pores of one specific size.

The description of ion transport in a membrane as an effective porous medium was undertaken by Mackie and Meares [51], Prager [52] and others. The membrane is represented as a structure containing a non-conductive backbone and a system of pores. They introduced the tortuosity factor; as well, several different equations for calculation of this 
parameter as a function of the volume fraction occupied by the polymer matrix impermeable to ion diffusion were proposed. Gluckauf [53] took into account the fact that the membrane contains pores of different size, introducing the function of pore distribution as a function of their radii. In his approach, diffusion permeability is considered as an average value for a medium in which the local value of the permeability coefficient is continuously changing in space. However, in the considered models, only the diffusion process is studied, but electromigration is not taken into account.

The microheterogeneous ( $\mathrm{MH}$ ) model [54] takes into account the presence of pores of different sizes. The membrane parts containing micropores form a gel phase considered as a homogeneous medium. The elements of the gel phase are separated from each other by intergel spaces. These intergel spaces are filled with the electrically neutral solution being in equilibrium with a local fragment of the membrane. They are interpreted as macropores and central parts of mesopores outside the electrical double layer on the pore walls. In the $\mathrm{MH}$ model both the diffusion and electromigration are taken into account. Application of the $\mathrm{MH}$ model for a large number of homogeneous and heterogeneous membranes has shown that it adequately describes the concentration dependences of conductivity, diffusion permeability and transport numbers [47]. The analysis made above shows that for describing the effects caused by the modification of ion-exchange membranes, carried out in Ref. [39], the application of microheterogeneous model seems the most appropriate.

In our work, we apply this model to study such effects, i.e. the influence of ion exchange membrane modification [39] on its conductivity, diffusion permeability and ion transport numbers. While the main idea of this modification was clogging the macropores, other treatments giving positive effect on the membrane permselectivity were performed. We will describe the responses of the membrane structure to these treatments through changes in the parameters of the microheterogeneous model. For the first time, we take into account that the intergel spaces are not completely filled with the aqueous solution, but partially with the PFSI material.

\section{Experimental}

For modeling, we use the experimental results obtained by Sarapulova et al. [39], who modified the CJMA-7 (Hefei Chemjoy Polymer Materials Co. Ltd., China) anion-exchange membrane (AEM) with a PFSI. The modification process went through several stages. In order to reasonably describe the effect of each of the stages, we will briefly present the modification procedure.

\subsection{Membrane and modifier}

The ion-exchange matrix of CJMA-7 membrane contains polyolefin functionalized with quaternary ammonium groups [55]. The matrix is crosslinked through the side chains [56]. This membrane is produced by the casting method and is reinforced with polyethylene terephthalate cloth by hot rolling.

An isopropyl alcohol solution of a perfluorosulfonated ionomer (PFSI) LF-4SK (JSC Plastopolimer, St. Petersburg, Russia), which is a liquid precursor of the MF-4SK (JSC Plastopolimer, St. Petersburg, Russia) membrane, was used for the CJMA-7 membrane modification. This polymer is an analogue of the Nafion ${ }^{\mathrm{TM}}$ material. Note that when preparing a membrane using an PFSI solution by the solution-casting method, the obtained membrane has a rather loose structure with a relatively high water content [57]. For this reason, for characterizing the PFSI material used in the modification [39], we have chosen the parameters of an MF-4SK membrane with a water content equal to $36.5 \mathrm{~mol} \mathrm{H} 2 \mathrm{O} / \mathrm{mol}$ fixed groups reported by Berezina et al. [58].

Some of the relevant characteristics of CJMA-7 membrane and the modifier are given in Table 1.

Table 1. Some characteristics of studied membrane 


\begin{tabular}{|c|c|c|c|c|c|}
\hline Membrane & Fixed groups & $\begin{array}{c}\text { Thickness in } \\
0.02 \mathrm{M} \mathrm{NaCl}, \\
\mu \mathrm{m}\end{array}$ & 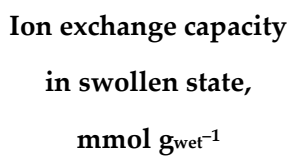 & $\begin{array}{c}\text { Water content, } \\
\text { mol } \mathrm{H}_{2} \mathrm{O} / \text { mol fixed } \\
\text { groups }\end{array}$ & $\begin{array}{l}\text { Density } \\
\text { in swollen state, } \\
\mathrm{g} \mathrm{cm}^{-3}\end{array}$ \\
\hline \multicolumn{6}{|c|}{ Mainly } \\
\hline CJMA-7 & $-N^{+}\left(\mathrm{CH}_{3}\right)_{3}$ & $174 \pm 10$ & $0.75 \pm 0.05$ & $21.7 \pm 1.0$ & $1.13 \pm 0.05$ \\
\hline PFSI & $-\mathrm{SO}_{3}^{-}$ & - & $0.87 \pm 0.05$ a & $36.5 \pm 1.0$ a & $1.40 \pm 0.15 \mathrm{~b}$ \\
\hline
\end{tabular}

a Data taken from [58]

b Data for a Nafion material, an analogue of LF-4SK, are taken from [59]

\subsection{Membrane modification}

The CJMA-7 membrane was modified in several stages:

1) a sample of a pristine CJMA-7 membrane (CJMA-7pr) was kept in a fridge for a year at a temperature of $4-6^{\circ} \mathrm{C}$ in a 0.1 eq $\mathrm{L}^{-1} \mathrm{NaCl}$ solution (CJMA-7fr); after that the obtained membrane was divided in two samples;

2) one of the samples was poured with a 7\% PFSI solution (perfluorinated sulfonated polymer in isopropyl alcohol) and dried at room temperature for $24 \mathrm{~h}$;

3 ) then the obtained membrane was divided into two more samples; the both samples were dried for an extra $1 \mathrm{~h}$ : the first one at $25^{\circ} \mathrm{C}\left(\mathrm{CJMA}-7_{\mathrm{fr}-\mathrm{mod} 25)}\right.$ and the second one at 50 ${ }^{\circ} \mathrm{C}$ (CJMA-7fr-mod50) in a drying oven.

Note that when the PFSI solution was poured on the surface of the CJMA-7 fr membrane, it passed through the membrane so that some amount of this solution appeared on the opposite (down) membrane side. With that, a thin film with a thickness of about $4 \mu \mathrm{m}$ was formed on the upper surface of the membrane [39].

Along with the CJMA-7fr-mod25 and CJMA-7fr-mod50 samples, the CJMA-7pr and CJMA$7 \mathrm{fr}$ samples were characterized. To equalize the conditions of preliminary preparation of the treated membranes, the CJMA-7 fr sample was kept in air at room temperature $\left(25^{\circ} \mathrm{C}\right)$ during $25 \mathrm{~h}$. For the above four membranes (CJMA-7pr, CJMA-7fr, CJMA-7fr-mod25 and CJMA-7fr-mod50), the concentration dependencies of membrane conductivity, diffusion permeability and transport numbers were determined. The details of the experiments are described in [39].

\subsection{Membrane characterization}

The membrane conductivity $\left(\kappa^{*}\right)$ was determined through the measurement of the membrane resistance by the differential method using a clip cell [60] at an alternating current frequency of $1 \mathrm{kHz}$. The integral diffusion permeability coefficient $(P)$ was obtained using a two-compartment flow cell where an electrolyte solution $\left(1000 \mathrm{~cm}^{3}\right)$ with a given concentration was circulated from one side of the membrane, and initially distilled water $\left(100 \mathrm{~cm}^{3}\right)$, from the other side [61]. The solute flux density $\left(J_{s}\right)$ through the membrane was determined by the rate of the solute concentration growth in the stream of initially distilled water. The value of $P$ was calculated as follows:

$$
P=\frac{J_{s} d_{m}}{C_{s}}
$$

where $d_{m}$ is the membrane thickness, $C_{s}$ is the solute concentration in the stream of the feed solution. The solute concentration in the dilute solution (initially distilled water) was found via the conductivity measurements [39]. The differential $\left(P^{*}\right)$ and integral permeability coefficients are linked as follows $[54,62]$ 


$$
P=\left(\int_{0}^{C_{s}} P^{*}(C) d C\right) / C_{s}, \quad P^{*}=P(1+\beta)
$$

where $\beta=d \log P / d \log C_{s}$.

Thus, the value of $P^{*}$ can be obtained from the concentration dependence of $P$ presented in bi-logarithmic coordinates $\left(\log P\right.$ vs. $\left.\log C_{s}\right)$.

The coion transport number was calculated using the following equations [63]

$$
t_{A}^{*}=\frac{F^{2}}{2 R T} \frac{P^{*} C_{s}}{\kappa^{*} t_{1}^{*}}, t_{1}^{*}+t_{A}^{*}=1
$$

where $F, R$ and $T$ are the Faraday, Gas constants and the temperature, respectively. The condition $t_{1}^{*}+t_{A}^{*}=1$ allows one to find the transport numbers of both the counterion (index " 1 ") and coion (index " $A$ ") using Eqs. (3).

\section{Theoretical}

The microheterogeneous model $(\mathrm{MH})$ [54] provides a rather simple description of membrane transport characteristics as functions of a single set of 6 parameters, which include two thermodynamic parameters (the ion-exchange capacity of the gel phase $(\bar{Q})$ and the Donnan equilibrium constant $\left(K_{D}\right)$ ); two structural parameters (the volume fractions of the gel phase $\left(f_{1}\right)$ and intergel spaces $\left(f_{2}, f_{1}+f_{2}=1\right)$, and a parameter $(\alpha)$, which reflects the relative disposition of the gel and solution phases); and two kinetic parameters (the ion diffusion coefficients $\left(\bar{D}_{i}\right)$ in the gel phase, $i=$ " + " and " - " for the cation $\left(\mathrm{Na}^{+}\right.$ion) and anion $\left(\mathrm{Cl}^{-}\right.$ion), respectively). In addition, the intergel solution is characterized also by ion diffusion coefficients $\left(D_{i}\right)$. In its conventional version, the model assumes that the intergel solution is identical to the external equilibrium solution, hence, $D_{i}$ do not differ from the ion diffusion coefficients in free solution. Parameter $\alpha$ can change between -1 and $+1: \alpha=-1$ refers to the case where the elements of the gel and solution phases are connected in series; $\alpha=+1$ refers to the case where these elements are connected in parallel.

The membrane conductivity $\left(\kappa^{*}\right)$, diffusion permeability $\left(P^{*}\right)$ and ion transport numbers $\left(t_{i}^{*}\right)$ in the membrane can be calculated through the effective membrane conductance coefficients, $L_{i}^{*}[54]$ :

$$
\begin{gathered}
\kappa^{*}=\left(z_{+}^{2} L_{+}^{*}+z_{-}^{2} L_{-}^{*}\right) F^{2} \\
P^{*}=R T\left(z_{+} L_{+}^{*} t_{-}^{*}+\left|z_{-}\right| L_{-}^{*} t_{+}^{*}\right) / c \\
t_{i}^{*}=z_{i}^{2} L_{i}^{*} /\left(z_{+}^{2} L_{+}^{*}+z_{-}^{2} L_{-}^{*}\right)=z_{i}^{2} L_{i}^{*} F^{2} / \kappa^{*}, i=+,-
\end{gathered}
$$

where $c=\left|z_{i}\right| c_{i}$ is the equivalent electrolyte concentration in the intergel solution (expressed in eq $\mathrm{L}^{-1}$ ). Expressions (4) and (5) are deduced from the irreversible thermodynamics using also the effective medium theory approach [64].

According to the MH model, the effective coefficients $L_{i}^{*}$ in the membrane are found through the ion conductance coefficients in the individual constituent phases:

$$
L_{i}^{*}=\left[f_{1}\left(\bar{L}_{i}\right)^{\alpha}+f_{2}\left(L_{i}\right)^{\alpha}\right]^{1 / \alpha}
$$

where $\bar{L}_{i}$ refers to the gel phase, and $L_{i}$, to the intergel electroneutral solution.

When describing the transport in the gel phase, we apply the assumptions of the Teorell-Meyer-Sievers (TMS) model. The gel phase is considered as homogeneous, the Nernst-Planck equation and the local electroneutrality condition are used there as well as 
in the intergel solution. $L_{i}$ and $\bar{L}_{i}$ are expressed as functions of the ionic diffusion coefficients, $D_{i}$ and $\bar{D}_{i}$, and the concentrations, $c_{i}$ and $\bar{c}_{i}$ in the corresponding phase [54]:

$$
L_{i}=D_{i} c_{i} / R T, \bar{L}_{i}=\bar{D}_{i} \bar{c}_{i} / R T
$$

Since locally, the gel phase and intergel solution are assumed in equilibrium, concentrations $c_{i}$ and $\bar{c}_{i}$ in these phases are linked by the Donnan relation:

$$
\begin{gathered}
\frac{\left(\bar{c}_{-}\right)^{1 / z_{-}}}{\left(\bar{c}_{+}\right)^{1 / z_{+}}}=K_{D} \frac{\left(c_{-}\right)^{1 / z_{-}}}{\left(c_{+}\right)^{1 / z_{+}}} \text {for an AEM, } \\
\frac{\left(\bar{c}_{+}\right)^{1 / z_{+}}}{\left(\bar{c}_{-}\right)^{1 / z_{-}}}=K_{D} \frac{\left(c_{+}\right)^{1 / z_{+}}}{\left(c_{-}\right)^{1 / z_{-}}} \text {for a cation-exchange membrane or PFSI material }
\end{gathered}
$$

The concentration of coions in the gel phase can be found when solving Equation (9) together with the electroneutrality condition in the gel phase, Equation (10):

$$
z_{-} \bar{c}_{-}+z_{+} \bar{c}_{+}=\bar{Q}
$$

The exchange capacity of the gel phase $(\bar{Q})$ is linked to the ion-exchange capacity of the membrane $(Q)$ as follows: $\bar{Q}=Q / f_{1}$.

\section{Results and discussion}

Figure 1 shows the experimental and simulated concentration dependencies of conductivity, diffusion permeability and counterion transport number for the membranes under study. As it can be seen, there is a good agreement, when the MH model parameters presented in Table 2 are used. Each stage of the membrane processing is reflected by a change in the parameters. Below we discuss the variations in the membrane structure occurring due to the treatment of the pristine membrane at each stage of its modification. As well, the corresponding changes in the model parameters are considered.

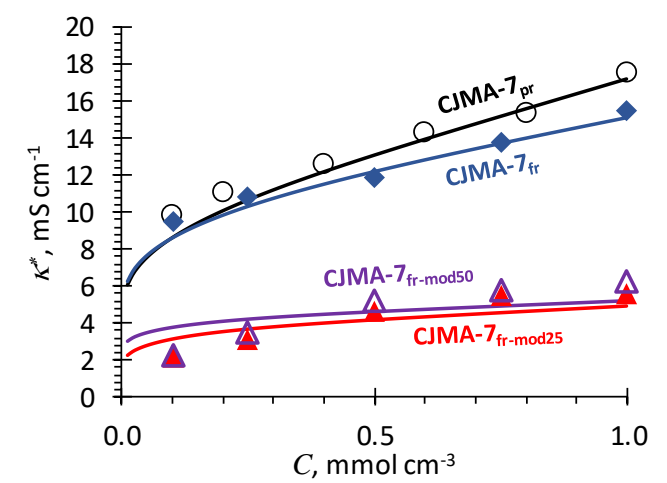

(a)

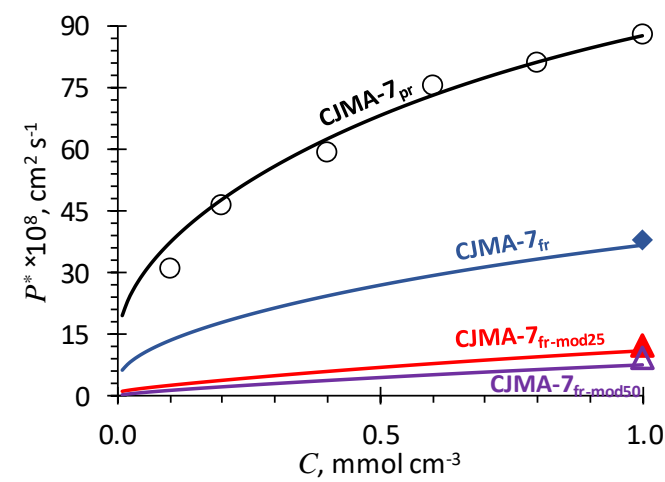

(b) 


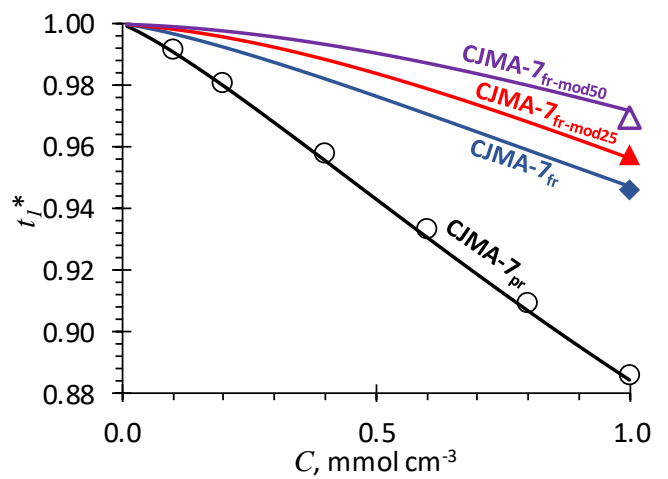

(c)

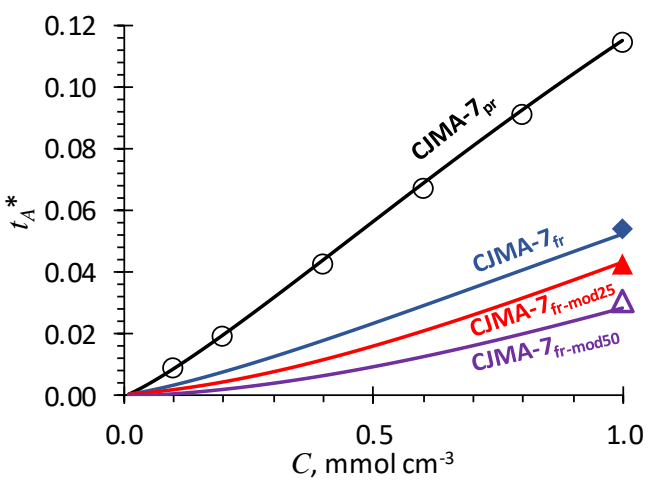

(d)

Figure 1. Experimental and simulated using the MH model concentration dependencies of (a) conductivity, (b) diffusion permeability, (c) counterion and (d) coion transport numbers for studied membrane samples (indicated near the corresponding curve). Markers denote the experimental data, lines denote the results of the simulation.

Table 2. Parameters of the MH model for the studied membrane samples in NaCl solution. PFSI-25 and PFSI-50 denote the PFSI material in the cases where the modified membrane was dried at $25^{\circ} \mathrm{C}$ and $50{ }^{\circ} \mathrm{C}$, respectively.

\begin{tabular}{|c|c|c|c|c|c|c|c|c|c|}
\hline & $f_{1}$ & $f_{2}$ & $\begin{array}{l}\bar{D}_{-} \times 10^{6}, \\
\mathrm{~cm}^{2} \mathrm{~s}^{-1}\end{array}$ & $\begin{array}{l}\bar{D}_{+} \times 10^{6}, \\
\mathrm{~cm}^{2} \mathrm{~s}^{-1}\end{array}$ & $\begin{array}{l}D_{-} \times 10^{6}, \\
\mathrm{~cm}^{2} \mathrm{~s}^{-1}\end{array}$ & $\begin{array}{l}D_{+} \times 10^{6}, \\
\mathrm{~cm}^{2} \mathrm{~s}^{-1}\end{array}$ & $\alpha$ & $K_{D}$ & $\begin{array}{c}\bar{Q} \\
\mathrm{mmol} \mathrm{cm} \mathrm{cm}^{-3}\end{array}$ \\
\hline CJMA-7pr & 0.82 & 0.18 & 2.30 & 1.50 & & & 0.30 & 0.07 & 0.92 \\
\hline CJMA-7fr & 0.84 & 0.16 & 2.25 & 0.65 & & & 0.25 & 0.05 & 0.94 \\
\hline CJMA-7fr-mod25 & 0.85 & 0.15 & 1.50 & 0.55 & 20.3 & 13.4 & 0.12 & 0.05 & 0.95 \\
\hline CJMA-7fr-mod50 & 0.90 & 0.10 & 1.45 & 0.50 & & & 0.10 & 0.05 & 1.0 \\
\hline PFSI-25 & - & - & - & - & 4.5 & 5.5 & - & 0.1 & 0.13 \\
\hline PFSI-50 & - & - & - & - & 4.3 & 5.2 & - & 0.1 & 0.18 \\
\hline
\end{tabular}

\subsection{Exposure to a low temperature}

As mentioned in Section 2.2, a sample of the pristine membrane, CJMA-7pr, was kept in a fridge at $4-6{ }^{\circ} \mathrm{C}$ for 12 months. Figure 1a shows, that the conductivity of CJMA-7fr decreased up to $12 \%$ as compared to the conductivity of CJMA-7pr; the conductivity changed more strongly in the range of high concentrations. With that, the diffusion permeability decreased by $57 \%$ at $C_{s}=1 \mathrm{~mol} \mathrm{~L}^{-1}$, the only concentration at which the measurement of $P^{*}$ was performed in Ref. [39]. Since, in the first approximation, the coion transport number, $t_{A}^{*}$, is proportional to $P^{*} / \kappa^{*}$ (Equation (3)), a stronger decrease in $P^{*}$ as compared to a decrease in $\kappa^{*}$ results in a significant decrease in $t_{A}^{*}$.

The fact that the diffusion permeability decreased when the membrane was maintained for a long period at low temperature, could be explained by the reorientation of the chains of the weakly cross-linked polymer forming the membrane matrix. According to Refs. [65-67], such a reorientation at low temperatures leads to a more compact packing of polymer chains, which results in a narrowing of the pores (Figure 2b). With decreasing 
temperature, the polymer structure becomes less loose, the free volume decreases [67]. Note, that the relaxation of an ion exchanger's polymer matrix during exposure to a low temperature takes quite a long time, for example, in the case of Nafion material, this time exceeds 1000 hours [68]. In the terms of the $\mathrm{MH}$ membrane parameters, these changes in the membrane structure under a low temperature can be interpreted as an increase in the volume fraction of the microporous gel phase, $f_{1}$, and a corresponding decrease in the volume fraction $f_{2}$ of intergel spaces (including the electroneutral solution in the central part of macro- and mesopores). This change in the values of the $f_{1}$ and $f_{2}$ parameters when converting CJMA-7 $\mathrm{pr}_{\mathrm{pr}}$ CJMA-7 $\mathrm{fr}$ is presented in Table 2. Another result of the narrowing of pores (namely of the micropores) is a decrease in the ionic diffusion coefficients in the gel phase. Recall that the diffusion coefficients in the electroneutral solution located in the macro- and mesopores are assumed the same as in the free solution. However, decreasing space in the micropores impedes the ion mobility. Moreover, the diffusion coefficient of coion should decrease more than that of counterion (Table 2). This is explained by the fact that a reduction in the distance between opposite pore walls leads to a decrease in the thickness of the electrically neutral or weakly charged solution located between two electrical double layers (EDLs) adjacent to the pore walls. This weakly charged solution is a pathway for coions through a narrow channel connecting two relatively large pores (ion clusters) (Figure 2). The EDLs are almost impermeable to coions because of the effect of Donnan coion exclusion: repelling of coions by the electrostatic force acting between them and the fixed positively charged functional groups [1]. The narrowing of micropores make some of them completely impermeable to coions, so they have to get around some regions of the gel phase. Therefore, the resulting pathway for coions became longer and more tortuous: compare Figures $2 \mathrm{a}$ and $2 \mathrm{~b}$. Since the $\mathrm{MH}$ model does not apply the tortuosity factor, we take into account the longer pathway of coions in the gel phase of CJMA-7fr by decreasing the diffusion coefficient of the coion $\left(\bar{D}_{+}\right)$to a greater extent than that of the counterion $\left(\bar{D}_{-}\right)$(Table 2$)$.

\section{Pristine}

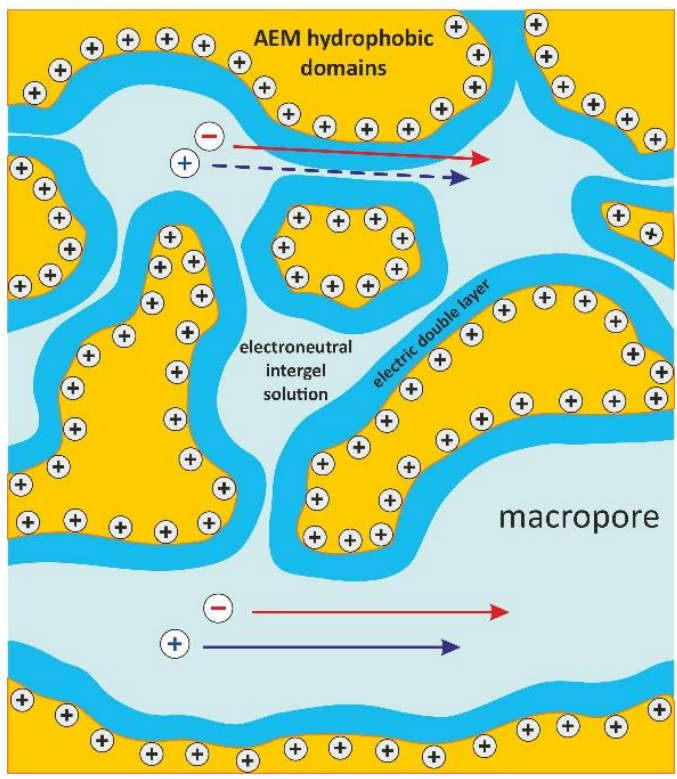

(a)
After long-term exposure to low temperature

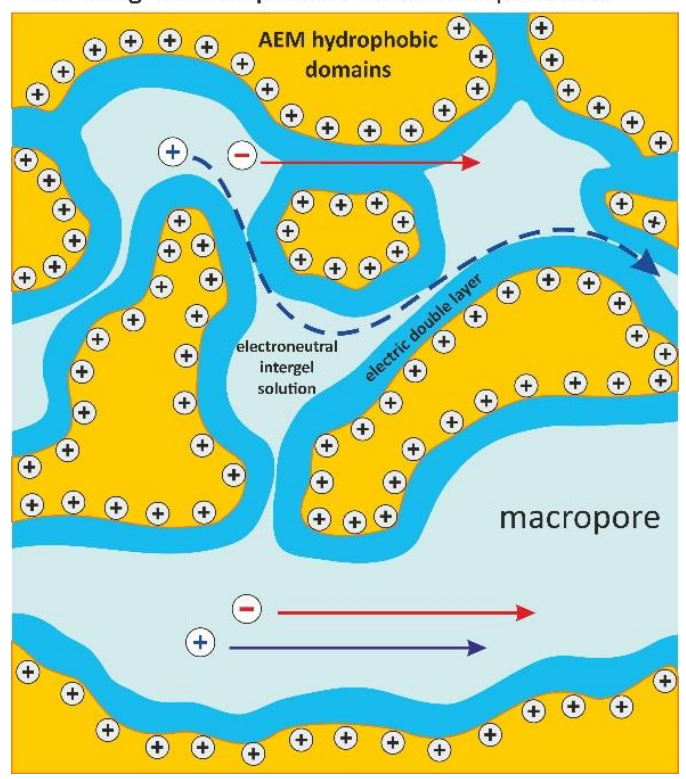

(b) 


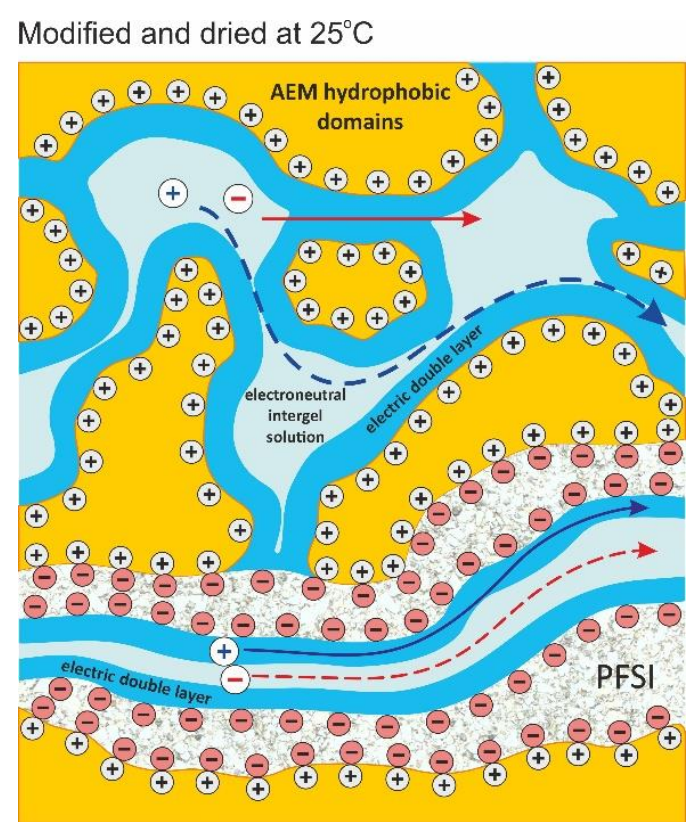

(c)

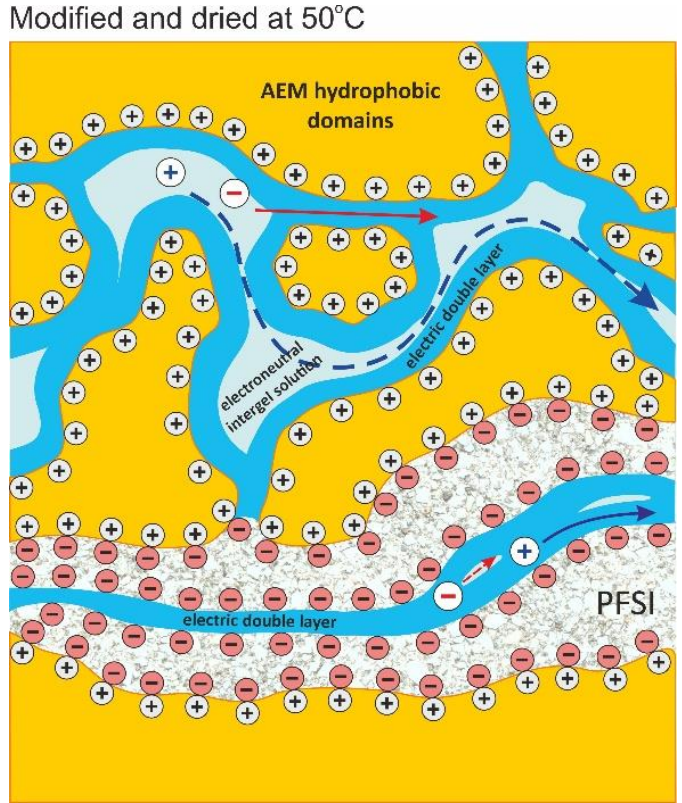

(d)

Figure 2. Schematic representation of the changes in studied membrane structure at different stages of its treatment: a) CJMA-7pr, b) CJMA-7fr, c) CJMA-7fr-mod25 and d) CJMA-7fr-mod50. The membrane structure is presented in accordence with modern concepts [69-71]: mesopores (ion clusters) are connected to each other by narrower ion-conducting channels; the macropores, which penetrate these finely porous areas, are due to structural defects including the gaps between ion-exchange material and non-conductive fillers, such as fibers of reinforcing cloth [43]. The arrows show ion fluxes during salt diffusion through the membrane. An animation that combines figures a-d is presented in Supplementary materials.

Due to a more compact structure, the concentration of fixed functional groups in the gel phase, $\bar{Q}$, slightly increases when passing from CJMA-7pr to CJMA-7fr, and the Donnan constant, $K_{D}$, decreases (Table 2). Since some domains of the gel phase becomes nonpermeable for coions, the contribution of the ion transport in parallel ways through gel phase and intergel solution increases, hence, the value of the $\alpha$ parameter decreases.

\subsection{Effect of membrane modification with PFSI solution}

Modification of CJMA-7fr membrane with PFSI (see CJMA-7 fr-mod25 or CJMA-7fr-mod50 in Figure 1) results in a dramatic decrease (almost by $60 \%$ ) in the conductivity as compared to CJMA-7 fr. At the same time, the diffusion permeability of the modified samples decreased even more significantly (up to $75 \%$ ), which led to a noticeable decrease in the coion transport numbers: by about $20 \%$ in the case of drying at $25{ }^{\circ} \mathrm{C}$ (the CJMA-7fr-mod25) and by $40 \%$ in case of drying at $50{ }^{\circ} \mathrm{C}$ (the CJMA- $7_{\text {fr-mod } 50)}$.

As it was mentioned above, there are two changes in the membrane structure due to this modification. First, a thin film (about 4 microns) of PFSA form on the membrane upper side (Section 2.2). Second, the PFSA solution penetrates into and through the large membrane pores. It follows from the experimental observation that some amount of this solution passes through the membrane when it is poured on the upper side of the CJMA- $7 \mathrm{fr}$ membrane [39]. The electrostatic interactions between the $-\mathrm{SO}^{-}$fixed groups of the PFSI and $-\mathrm{N}^{+}\left(\mathrm{CH}_{3}\right)_{3}$ groups of the substrate membrane facilitate the penetration of the PFSI into macropores. However, it is not likely that it fills them completely. Even if the PFSI solution occupies the entire pore volume, after evaporation of the solvent (isopropyl alcohol), the PFSI volume decreases. 
Let us evaluate the effect of the PFSI film on the membrane conductivity and diffusion permeability.

If we neglect the concentration dependence of the membrane diffusion permeability and conductivity, the integral coefficient of diffusion permeability $\left(P_{t o t}\right)$ and conductivity $\left(\kappa_{\text {tot }}\right.$ ) of the modified bilayer membrane can be expressed as:

$$
P_{\text {tot }}=\left(d_{\text {sub }}+d_{\text {film }}\right)\left(\frac{d_{\text {sub }}}{P_{\text {sub }}}+\frac{d_{\text {film }}}{P_{\text {film }}}\right)^{-1} \text { and } \kappa_{\text {tot }}=\left(d_{\text {sub }}+d_{\text {film }}\right)\left(\frac{d_{\text {sub }}}{\kappa_{\text {sub }}}+\frac{d_{\text {film }}}{\kappa_{\text {film }}}\right)^{-1}
$$

where $d$ is the thickness of one of the layers (the substrate or film); indices "sub" and "film" denotes the substrate (the CJMA-7 fr membrane) and a film, respectively.

As it can be seen from Equation (12), due to a very small value of the ratio $d_{\text {film }} / d_{\text {sub }}$ (equal to $4 / 174=0.023$ ) in the case of a relatively thin modifying film, and relatively close values of $P_{s u b}$ and $P_{t o t}$, as well as $\kappa_{\text {sub }}$ and $\kappa_{t o t}$, the resulting parameters of the bilayer membrane and substrate are quite close to each other. For example, in $1 \mathrm{M} \mathrm{NaCl}$ solution, the CJMA-7 fr membrane parameters are $P_{\text {sub }}=38 \times 10^{-8} \mathrm{~cm}^{2} \mathrm{~s}^{-1}$ and $\kappa_{\text {sub }}=15.5 \mathrm{mS} \mathrm{cm}^{-1}$. For the PFSI in the same solution, $P_{\text {film }}=12.9 \times 10^{-8} \mathrm{~cm}^{2} \mathrm{~s}^{-1}$ and $\kappa_{\text {film }} \approx 8 \mathrm{mS} \mathrm{cm}^{-1}$ (the data are taken for a MF-4SK membrane [72]). Calculations using Equation (12) yields $P_{\text {tot }}=$ $36.4 \times 10^{-8} \mathrm{~cm}^{2} \mathrm{~s}^{-1}$ and $\kappa_{t o t}=15.2 \mathrm{mS} \mathrm{cm}^{-1}$. The decrease in $P_{t o t}$ and $\kappa_{t o t}$ observed in the experiments [39] (Figure 1a,b) when passing from the CJMA-7 fr membrane to the CJMA7 fr-mod25 membrane is much greater $\left[\left(P_{t o t} / P_{\text {sub }}\right)_{\exp } \approx 3.2\right.$ and $\left.\left(\kappa_{\text {tot }} / \kappa_{\text {sub }}\right)_{\exp } \approx 2.5\right]$ than the above estimations show. Therefore, we can neglect the presence of a thin PFSI film on the modified membrane surface, and take into account only the following two effects: (1) the penetration of the PFSI into the macropores of the substrate membrane, and (2) drying of the composite membrane at an elevated temperature.

1) The fact that the liquid PFSI passes through the CJMA-7 fr membrane suggests that the PFSI material occupies only a part of the macropores, so that a space available for solution remains in the pore center (Figure 2c). The assumption that this material covers the pore walls follows from the presence of negatively charged fixed groups $\left(-\mathrm{SO}_{3}^{-}\right)$in it. These groups can interact electrostatically with the positively charged fixed groups ( $\left.-\mathrm{N}^{+}\left(\mathrm{CH}_{3}\right)_{3}\right)$ in the CJMA-7 membrane. Two oppositely charged functional groups can be quite close to each other since PFSI is a liquid. They tend to neutralize their charges, however, the groups still have an attraction to cations and anions and tend to associate with them also [73]. It can be assumed that the $-\mathrm{SO}_{3}^{-}$fixed groups in thin nanometer layer of PFSI would be neutralized with the $-\mathrm{N}^{+}\left(\mathrm{CH}_{3}\right)_{3}$ groups of the CJMA-7 membrane located on the macropore walls. However, the PFSI material retains its functional groups at some distance from the pore walls. Even if a half of the functional groups of PFSI will be neutralized, the relative amount of the neutralized positively charged groups of the CJMA-7 membrane will be small compared to the total amount of this groups.

2) When drying, the polymer loses water, which leads to a shrinkage of membrane matrix and narrowing the pores. As a result, $f_{1}$ increases ( $f_{2}$ decreases). In addition, the ion diffusion coefficients in the gel phase decrease (parameters $\bar{D}_{-}$and $\bar{D}_{+}$). The higher the drying temperature, the greater changes in the above parameters (Table 2). The decrease in water content leads also to an increase in the gel exchange capacity $\bar{Q}$ (expressed in mmol $\mathrm{cm}^{-3}$ ) and a decrease in $\alpha$ (Table 2). As for the modelling of the contribution of the PFSI in the macropores, it is made by calculation of the $L_{+}$and $L_{-}$parameters (related to the intergel domains) using Eqs. (8). These parameters are calculated as for a homogeneous medium consisted of PFSI and an aqueous solution. In the case of $25^{\circ} \mathrm{C}$ of drying temperature, the diffusion coefficients $D_{+}$and $D_{-}$are taken $20 \%$ greater than those found 
for a strongly watered MF-4SK membrane (with water content $36.5 \mathrm{~mol} \mathrm{H} \mathrm{H}_{2} \mathrm{O} / \mathrm{mol}$ functional groups, Table 1). The values of $D_{+}$and $D_{-}$are set lower in the case of $50^{\circ} \mathrm{C}$, in the same proportion as for the membrane-substrate. The concentrations of cations and anions are calculated using the Donnan equation, Equation (10), applied as for a cation-exchange membrane. However, the value of exchange capacity of the material filling the intergel domains, $\bar{Q}$, is taken rather low, when assuming that PFSI fills 33\% and $50 \%$ of the intergel space volume in the cases of $25^{\circ} \mathrm{C}$ and $50^{\circ} \mathrm{C}$ of the drying temperature, respectively. Since more water leaves the membrane when drying at $50^{\circ} \mathrm{C}$, the $\bar{Q}$ value for both membrane gel phase and intergel domains is set greater at this temperature than at $25^{\circ} \mathrm{C}$.

As Figure 2 shows there is a good agreement between computational results and experimental data. We would like to show that each stage of membrane treatment can be described by a reasonable change of the membrane structural and kinetic parameters of the MH model. We discussed above only the direction of the parameters' changes; their values were found by fitting the experimental data. However, the response of the parameter values to one or another method of membrane modification makes it possible to better understand what consequences this method has. In particular, when we consider Equation (3), we see that in order to increase the membrane permselectivity towards the counterion transport, we have to decrease the value of $P^{*}$ and increase that of $\kappa^{*}$ : the membrane diffusion permeability, $P^{*}$, is controlled by the transport of coions, and the conductivity, $\kappa^{*}$, by the transport of counterions. Nevertheless, when changing some condition, usually both parameters vary in the same direction. For example, when decreasing water content (e.g., by drying the membrane at an elevated temperature), the mobilities of both counterion and coion decreases. With that, the effective mobility of coion decreases in a greater extent, since the pathway of this ion increases, as explained above. Therefore, this makes it possible to increase the membrane permselectivity, although at the expense of a decrease in the membrane conductivity. This is an example of the trade-off between membrane selectivity and permeability/conductivity, which is intensively discussed in the literature $[33,38,74]$. As it is shown in our paper, "clogging" the macropores with a polymer is a promising way to improve this trade-off towards higher selectivity without significant loss of conductivity.

\section{Conclusion}

The microheterogeneous model with some new amendments is applied to simulate the changes in membrane structure and related transport properties due to different modifications of an anion-exchange CJMA-7 membrane. The casting of a perfluorosulfonated ionomer solution on the CJMA-7 membrane surface resulted in 1) formation of a thin (4 $\mathrm{mm}$ ) PFSI film on the surface of the membrane substrate, and 2) partial filling of the substrate macropores with the PFSI material. The macropores are mainly due to the gaps between relatively homogeneous ion-exchange material and non-conductive fillers, such as fibers of reinforcing cloth.

The description of the experimental results of this modification is done earlier by Sarapulova et al. [39]. The experiment has been shown that after several stages of modification of the CJMA-7 membrane, the coion ( $\mathrm{Na}^{+}$) transport number in the composite membrane equilibrated with $1 \mathrm{M} \mathrm{NaCl}$ solution decreased from 0.11 to 0.03 .

The modeling shows that the effect of the additional film is negligible due to its thin thickness, and relatively close transport characteristics of the film and substrate.

Due to the interactions between the $-\mathrm{SO}_{3}{ }^{-}$fixed groups of the PFSI and $-\mathrm{N}^{+}\left(\mathrm{CH}_{3}\right)_{3}$ groups of the substrate membrane, the PFSI enters the macropores and partly fills them. The main effect leading to a greater permselectivity of the modified membrane is partial blocking of macropores of the CJMA-7 membrane with the PFSI material. Thus, the coion transport across the membrane occurring mainly through the macropores is significantly reduced. Therefore, the membrane microporous gel phase having a high selectivity to counterions becomes the principal pathway for both counterions and coions. 
Acknowledgement: The authors thank Olesya Rybalkina for kindly providing experimental data on the conductivity and diffusion permeability of the CJMA-7 membrane.

Author Contributions: A.K.: Data analysis and curation; Draft preparation, writing and editing. N.P.: Methodology; Research design and investigation. V.V.: Research conceptualization and investigation; Manuscript writing, revision and validation; Funding acquisition; Project administration. All authors have read and agreed to the published version of the manuscript.

Funding: We are grateful to the Russian Foundation for Basic Research, project Nb 20-08-00933 A.

Conflicts of Interest: The authors declare no conflict of interest.

\section{References}

1. Helfferich, F. Ion Exchange; McGraw-Hill, New York, 1962;

2. Strathmann, H. Applications of Ion-Exchange Membrane Separation Processes. Membr. Sci. Technol. 2004, 9, 287330, doi:10.1016/S0927-5193(04)80037-8.

3. Strathmann, H. Electrodialysis, a mature technology with a multitude of new applications. Desalination 2010, 264 , 268-288, doi:10.1016/j.desal.2010.04.069.

4. Ran, J.; Wu, L.; He, Y.; Yang, Z.; Wang, Y.; Jiang, C.; Ge, L.; Bakangura, E.; Xu, T. Ion exchange membranes: New developments and applications. J. Memb. Sci. 2017, 522, 267-291, doi:10.1016/j.memsci.2016.09.033.

5. Zhang, X.; Liu, Y. Circular economy-driven ammonium recovery from municipal wastewater: State of the art, challenges and solutions forward. Bioresour. Technol. 2021, 334, 125231, doi:10.1016/J.BIORTECH.2021.125231.

6. Merle, G.; Wessling, M.; Nijmeijer, K. Anion exchange membranes for alkaline fuel cells: A review. J. Memb. Sci. 2011, 377, 1-35, doi:10.1016/j.memsci.2011.04.043.

7. Yaroslavtsev, A.B.; Dobrovolsky, Y.A.; Shaglaeva, N.S.; Frolova, L.A.; Gerasimova, E. V; Sanginov, E.A. Nanostructured materials for low-temperature fuel cells. Russ. Chem. Rev. 2012, 81, 191-220, doi:10.1070/rc2012v081n03abeh004290.

8. Wang, J.; Zhao, Y. Poly(aryl piperidinium) membranes and ionomers for hydroxide exchange membrane fuel cells. Nat. Energy, doi:10.1038/s41560-019-0372-8.

9. Thangarasu, S.; Oh, T.H. Progress in poly(phenylene oxide) based cation exchange membranes for fuel cells and redox flow batteries applications. Int. J. Hydrogen Energy 2021, 46, 38381-38415, doi:10.1016/J.IJHYDENE.2021.09.081.

10. Ye, J.; Cheng, Y.; Sun, L.; Ding, M.; Wu, C.; Yuan, D.; Zhao, X.; Xiang, C.; Jia, C. A green SPEEK/lignin composite membrane with high ion selectivity for vanadium redox flow battery. J. Memb. Sci. 2019, 572, 110-118, doi:10.1016/J.MEMSCI.2018.11.009.

11. Jiang, S.; Sun, H.; Wang, H.; Ladewig, B.P.; Yao, Z. A comprehensive review on the synthesis and applications of ion exchange membranes. Chemosphere 2021, 282, 130817, doi:10.1016/J.CHEMOSPHERE.2021.130817.

12. Luo, T.; Abdu, S.; Wessling, M. Selectivity of ion exchange membranes: A review. J. Memb. Sci. 2018, 555, 429454, doi:10.1016/j.memsci.2018.03.051.

13. Xu, S.; Song, J.; Bi, Q.; Chen, Q.; Zhang, W.M.; Qian, Z.; Zhang, L.; Xu, S.; Tang, N.; He, T. Extraction of lithium from Chinese salt-lake brines by membranes: Design and practice. J. Memb. Sci. 2021, 635, 119441, 
doi:10.1016/J.MEMSCI.2021.119441.

14. Merino-Garcia, I.; Velizarov, S. New insights into the definition of membrane cleaning strategies to diminish the fouling impact in ion exchange membrane separation processes. Sep. Purif. Technol. 2021, 277, 119445, doi:10.1016/J.SEPPUR.2021.119445.

15. Sharma, M.; Das, P.P.; Chakraborty, A.; Purkait, M.K. Clean energy from salinity gradients using pressure retarded osmosis and reverse electrodialysis: A review. Sustain. Energy Technol. Assessments 2022, 49, 101687, doi:10.1016/J.SETA.2021.101687.

16. Wenten, I.G.; Khoiruddin. Recent developments in heterogeneous ion-exchange membrane: Preparation, modification, characterization and performance evaluation. J. Eng. Sci. Technol. 2016, 11, 916-934.

17. Ariono, D.; Khoiruddin; Subagjo; Wenten, I.G. Heterogeneous structure and its effect on properties and electrochemical behavior of ion-exchange membrane. Mater. Res. Express 2017, 4, doi:10.1088/2053-1591/aa5cd4.

18. Kotoka, F.; Merino-Garcia, I.; Velizarov, S. Surface modifications of anion exchange membranes for an improved reverse electrodialysis process performance: A review. Membranes (Basel). 2020, 10, 1-22.

19. Kucera, J. Reverse Osmosis; John Wiley \& Sons, Inc.: Hoboken, NJ, USA, 2010; ISBN 9780470882634.

20. Kedem, O.; Schechtmann, L.; Mirsky, Y.; Saveliev, G.; Daltrophe, N. Low-polarisation electrodialysis membranes. Desalination 1998, 118, 305-314, doi:10.1016/S0011-9164(98)00153-2.

21. Andreeva, M.A.; Gil, V.V.; Pismenskaya, N.D.; Nikonenko, V.V.; Dammak, L.; Larchet, C.; Grande, D.; Kononenko, N.A. Effect of homogenization and hydrophobization of a cation-exchange membrane surface on its scaling in the presence of calcium and magnesium chlorides during electrodialysis. J. Memb. Sci. 2017, 540, 183-191, doi:10.1016/J.MEMSCI.2017.06.030.

22. Sharafan, M.V.; Zabolotskii, V.I.; Bugakov, V.V. Electric mass transport through homogeneous and surfacemodified heterogeneous ion-exchange membranes at a rotating membrane disk. Russ. J. Electrochem. 2009, 45, 1162-1169, doi:10.1134/S1023193509100085.

23. Andreeva, M.A.; Gil, V.V.; Pismenskaya, N.D.; Dammak, L.; Kononenko, N.A.; Larchet, C.; Grande, D.; Nikonenko, V.V. Mitigation of membrane scaling in electrodialysis by electroconvection enhancement, $\mathrm{pH}$ adjustment and pulsed electric field application. J. Memb. Sci. 2018, 549, 129-140, doi:10.1016/j.memsci.2017.12.005.

24. Sata, T. Studies on anion exchange membranes having permselectivity for specific anions in electrodialysis Effect of hydrophilicity of anion exchange membranes on permselectivity of anions. J. Memb. Sci. 2000, 167, 131, doi:10.1016/S0376-7388(99)00277-X.

25. Zhu, Y.; Ahmad, M.; Yang, L.; Misovich, M.; Yaroshchuk, A.; Bruening, M.L. Adsorption of polyelectrolyte multilayers imparts high monovalent/divalent cation selectivity to aliphatic polyamide cation-exchange membranes. J. Memb. Sci. 2017, 537, 177-185, doi:10.1016/j.memsci.2017.05.043.

26. Wang, Z.; Liang, S.; Kang, Y.; Zhao, W.; Xia, Y.; Yang, J.; Wang, H.; Zhang, X. Manipulating interfacial polymerization for polymeric nanofilms of composite separation membranes. Prog. Polym. Sci. 2021, 122, 101450, doi:10.1016/J.PROGPOLYMSCI.2021.101450.

27. Zabolotskii, V.I.; Melnikov, S.S.; Achoh, A.R. Nitrate selective anion exchange membrane 140771 U1 2014. 
28. Chen, J.; Cao, J.; Zhang, R.; Zhou, J.; Wang, S.; Liu, X.; Zhang, T.; Tao, X.; Zhang, Y. Modifications on Promoting the Proton Conductivity of Polybenzimidazole-Based Polymer Electrolyte Membranes in Fuel Cells. Membranes (Basel). 2021, 11, 826, doi:10.3390/membranes11110826.

29. Agel, E.; Bouet, J.; Fauvarque, J.. Characterization and use of anionic membranes for alkaline fuel cells. J. Power Sources 2001, 101, 267-274, doi:10.1016/S0378-7753(01)00759-5.

30. Wilhelm, F.G.; Pünt, I.; van der Vegt, N.F.A.; Strathmann, H.; Wessling, M. Asymmetric Bipolar Membranes in Acid-Base Electrodialysis. Ind. Eng. Chem. Res. 2002, 41, 579-586, doi:10.1021/ie010524n.

31. Pärnamäe, R.; Mareev, S.; Nikonenko, V.; Melnikov, S.; Sheldeshov, N.; Zabolotskii, V.; Hamelers, H.V.M.; Tedesco, M. Bipolar membranes: A review on principles, latest developments, and applications. J. Memb. Sci. 2021, 617, 118538, doi:10.1016/j.memsci.2020.118538.

32. Melnikov, S.; Zabolotskii, V.; Sheldeshov, N.; Achoh, A.; Bondarev, D. Catalysis of water splitting reaction in asymmetric bipolar membranes with different chemical composition of cation-exchange layer. Desalin. WATER Treat. 2018, 124, 30-36, doi:10.5004/dwt.2018.22734.

33. Park, H.B.; Kamcev, J.; Robeson, L.M.; Elimelech, M.; Freeman, B.D. Maximizing the right stuff: The trade-off between membrane permeability and selectivity. Science (80-. ). 2017, 356, 1138-1148, doi:10.1126/science.aab0530.

34. Epsztein, R.; DuChanois, R.M.; Ritt, C.L.; Noy, A.; Elimelech, M. Towards single-species selectivity of membranes with subnanometre pores. Nat. Nanotechnol. 2020, 15, 426-436, doi:10.1038/s41565-020-0713-6.

35. Reig, M.; Farrokhzad, H.; Van der Bruggen, B.; Gibert, O.; Cortina, J.L. Synthesis of a monovalent selective cation exchange membrane to concentrate reverse osmosis brines by electrodialysis. Desalination 2015, 375, 1-9, doi:10.1016/j.desal.2015.07.023.

36. Ounissi, T.; Dammak, L.; Fauvarque, J.-F.; Selmane Bel Hadj Hmida, E. Ecofriendly lithium-sodium separation by diffusion processes using lithium composite membrane. Sep. Purif. Technol. 2021, 275, 119134, doi:10.1016/j.seppur.2021.119134.

37. Golubenko, D.V.; Karavanova, Y.A.; Melnikov, S.S.; Achoh, A.R.; Pourcelly, G.; Yaroslavtsev, A.B. An approach to increase the permselectivity and mono-valent ion selectivity of cation-exchange membranes by introduction of amorphous zirconium phosphate nanoparticles. J. Memb. Sci. 2018, 563, 777-784, doi:10.1016/j.memsci.2018.06.024.

38. Stenina, I.; Golubenko, D.; Nikonenko, V.; Yaroslavtsev, A. Selectivity of Transport Processes in Ion-Exchange Membranes: Relationship with the Structure and Methods for Its Improvement. Int. J. Mol. Sci. 2020, 21, 5517, doi:10.3390/ijms21155517.

39. Sarapulova, V.; Pismenskaya, N.; Titorova, V.; Sharafan, M.; Wang, Y.; Xu, T.; Zhang, Y.; Nikonenko, V. Transport characteristics of CJMAED ${ }^{\mathrm{TM}}$ homogeneous anion exchange membranes in sodium chloride and sodium sulfate solutions. Int. J. Mol. Sci. 2021, 22, 1-24, doi:10.3390/ijms22031415.

40. Kingsbury, R.S.; Coronell, O. Modeling and validation of concentration dependence of ion exchange membrane permselectivity: Significance of convection and Manning's counter-ion condensation theory. J. Memb. Sci. 2021, 620, 118411, doi:10.1016/j.memsci.2020.118411.

41. Luo, T.; Roghmans, F.; Wessling, M. Ion mobility and partition determine the counter-ion selectivity of ion 
exchange membranes. J. Memb. Sci. 2020, 597, 117645, doi:10.1016/j.memsci.2019.117645.

42. Akberova, E.M.; Vasil'eva, V.I.; Zabolotsky, V.I.; Novak, L. A Study of Ralex Membrane Morphology by SEM. Membranes (Basel). 2019, 9, 169, doi:10.3390/membranes9120169.

43. Sarapulova, V.; Shkorkina, I.; Mareev, S.; Pismenskaya, N.; Kononenko, N.; Larchet, C.; Dammak, L.; Nikonenko, $\mathrm{V}$. Transport characteristics of fujifilm ion-exchange membranes as compared to homogeneous membranes AMX and CMX and to heterogeneous membranes MK-40 and MA-41. Membranes (Basel). 2019, 9, 1-23, doi:10.3390/membranes9070084.

44. Kamcev, J.; Sujanani, R.; Jang, E.S.; Yan, N.; Moe, N.; Paul, D.R.; Freeman, B.D. Salt concentration dependence of ionic conductivity in ion exchange membranes. J. Memb. Sci. 2018, 547, 123-133, doi:10.1016/j.memsci.2017.10.024.

45. Filippov, A.N.; Koroleva, Y.O.; Verma, A.K. Cell Model of a Fibrous Medium (Membrane). Comparison between Two Different Approaches to Varying Liquid Viscosity. Membr. Membr. Technol. 2020, 2, 230-243, doi:10.1134/S2517751620040058.

46. Filippov, A.; Afonin, D.; Kononenko, N.; Lvov, Y.; Vinokurov, V. New approach to characterization of hybrid nanocomposites. Colloids Surfaces A Physicochem. Eng. Asp. 2017, 521, 251-259, doi:10.1016/j.colsurfa.2016.08.079.

47. Nikonenko, V.; Nebavsky, A.; Mareev, S.; Kovalenko, A.; Urtenov, M.; Pourcelly, G. Modelling of ion transport in electromembrane systems: Impacts of membrane bulk and surface heterogeneity. Appl. Sci. 2018, 9, doi:10.3390/app9010025.

48. Guzmán-Garcia, A.G.; Pintauro, P.N.; Verbrugge, M.W.; Hill, R.F. Development of a space-charge transport model for ion-exchange membranes. AIChE J. 1990, 36, 1061-1074, doi:10.1002/aic.690360713.

49. Peters, P.B.; van Roij, R.; Bazant, M.Z.; Biesheuvel, P.M. Analysis of electrolyte transport through charged nanopores. Phys. Rev. E 2016, 93, 053108, doi:10.1103/PhysRevE.93.053108.

50. Choy, T.C. Effective Medium Theory; Oxford University Press, 2015; ISBN 9780198705093.

51. Mackie, J.S.; Meares, P. The diffusion of electrolytes in a cation-exchange resin membrane I. Theoretical. Proc. R. Soc. London. Ser. A. Math. Phys. Sci. 1955, 232, 498-509, doi:10.1098/rspa.1955.0234.

52. Prager, S. Diffusion in Inhomogeneous Media. J. Chem. Phys. 1960, 33, 122-127, doi:10.1063/1.1731066.

53. Glueckauf, E.; A, P.R.S.L. A new approach to ion exchange polymers. Proc. R. Soc. London. Ser. A. Math. Phys. Sci. 1962, 268, 350-370, doi:10.1098/rspa.1962.0146.

54. Zabolotsky, V.I.; Nikonenko, V. V. Effect of structural membrane inhomogeneity on transport properties. J. Memb. Sci. 1993, 79, 181-198, doi:10.1016/0376-7388(93)85115-D.

55. Wang, Y.; Zhang, Z.; Jiang, C.; Xu, T. Recovery of gamma-aminobutyric acid (GABA) from reaction mixtures containing salt by electrodialysis. Sep. Purif. Technol. 2016, 170, 353-359, doi:10.1016/J.SEPPUR.2016.07.002.

56. Yan, H.; Wang, Y.; Xu, T. Developing Ion Exchange Membrane for Treating High Salinity Water Using Electrodialysis. In Proceedings of the Proceedings of the 5th international conferences on Sustainable Chemical Product and Process Engineering (SCPPE); Tianjin, China, 2019; pp. 65-67.

57. Berezina, N.P.; Timofeev, S. V.; Kononenko, N.A. Effect of conditioning techniques of perfluorinated sulphocationic membranes on their hydrophylic and electrotransport properties. J. Memb. Sci. 2002, 209, 509-518, 
doi:10.1016/S0376-7388(02)00368-X.

58. Berezina, N.P.; Kubaisi, A.A.R.; Alpatova, N.M.; Andreev, V.N.; Griga, E.I. Composite Polyaniline/MF-4SK Membranes: A Chemical Template Synthesis and the Sorption and Conduction Properties. Russ. J. Electrochem. 2004, 40, 286-293, doi:10.1023/B:RUEL.0000019666.97442.03.

59. Zook, L.A.; Leddy, J. Density and Solubility of Nafion: Recast, Annealed, and Commercial Films. Anal. Chem. 1996, 68, 3793-3796, doi:10.1021/ac960604e.

60. Lteif, R.; Dammak, L.; Larchet, C.; Auclair, B. Conductivitéélectrique membranaire: étude de l'effet de la concentration, de la nature de l'électrolyte et de la structure membranaire. Eur. Polym. J. 1999, 35, 1187-1195, doi:10.1016/S0014-3057(98)00213-4.

61. Pismenskaya, N.D.; Nevakshenova, E.E.; Nikonenko, V. V Using a Single Set of Structural and Kinetic Parameters of the Microheterogeneous Model to Describe the Sorption and Kinetic Properties of Ion-Exchange Membranes. Pet. Chem. 2018, 58, 147-156, doi:10.1134/S0965544118060087.

62. Gnusin, N.P.; Berezina, N.P.; Shudrenko, A.A.; Ivina, A.P. Electrolyte diffusion across ion-exchange membranes. Russ. J. Phys. Chem. A 1994, 68, 506-510.

63. Auclair, B.; Nikonenko, V.; Larchet, C.; Métayer, M.; Dammak, L. Correlation between transport parameters of ion-exchange membranes. J. Memb. Sci. 2002, 195, 89-102, doi:10.1016/S0376-7388(01)00556-7.

64. Nikonenko, V. V.; Yaroslavtsev, A.B.; Pourcelly, G. Ion Transfer in and Through Charged Membranes: Structure, Properties, and Theory. In Ionic Interactions in Natural and Synthetic Macromolecules; John Wiley \& Sons, Inc.: Hoboken, NJ, USA, 2012; pp. 267-335 ISBN 9780470529270.

65. Löwemark, L.; Liao, A.C.-Y.; Liou, Y.-H.; Godad, S.; Chang, T.-Y.; Kunz, A. Potential and pitfalls of XRF-CS analysis of ion-exchange resins in environmental studies. Sci. Rep. 2021, 11, 20941, doi:10.1038/s41598-021-004469.

66. Aittola, J.-P.; Chyssler, J.; Ringberg, H. Thermal stability of ion-exchange resins; Studsvik Energiteknik AB, 1982;

67. Perepechko, I. Viscoelastic parameters of polymers at low temperatures. In Low-Temperature Properties of Polymers; Elsevier, 1980; pp. 241-254 ISBN 978-0-08-025301-5.

68. Alberti, G.; Narducci, R.; Sganappa, M. Effects of hydrothermal/thermal treatments on the water-uptake of Nafion membranes and relations with changes of conformation, counter-elastic force and tensile modulus of the matrix. J. Power Sources 2008, 178, 575-583, doi:10.1016/j.jpowsour.2007.09.034.

69. Gierke, T.D.; Munn, G.E.; Wilson, F.C. The morphology in nafion perfluorinated membrane products, as determined by wide- and small-angle x-ray studies. J. Polym. Sci. Polym. Phys. Ed. 1981, 19, 1687-1704, doi:10.1002/pol.1981.180191103.

70. Kreuer, K.-D.; Paddison, S.J.; Spohr, E.; Schuster, M. Transport in Proton Conductors for Fuel-Cell Applications: Simulations, Elementary Reactions, and Phenomenology. Chem. Rev. 2004, 104, 4637-4678, doi:10.1021/cr020715f.

71. Mauritz, K.A.; Moore, R.B. State of Understanding of Nafion. Chem. Rev. 2004, 104, 4535-4586, doi:10.1021/cr0207123.

72. Larchet, C.; Dammak, L.; Auclair, B.; Parchikov, S.; Nikonenko, V. A simplified procedure for ion-exchange membrane characterisation. New J. Chem. 2004, 28, 1260-1267, doi:10.1039/b316725a. 
73. Rollins, C.; Jensen, L.; Schwartz, A.N. Desalting of Amino Acid Solutions by an Ion Retardation Resin. Anal. Chem. 1962, 34, 711-712, doi:10.1021/ac60186a038.

74. Shin, M.G.; Seo, J.Y.; Park, H.; Park, Y.-I.; Lee, J.-H. Overcoming the permeability-selectivity trade-off of desalination membranes via controlled solvent activation. J. Memb. Sci. 2021, 620, 118870, doi:10.1016/j.memsci.2020.118870. 